Neurosurg Focus 19 (6):E7, 2005

\title{
Endoscopic management of intracranial cysts
}

\author{
Jeffrey P. Greenfield, M.D., Ph.D., ANd Mark M. Souweidane, M.D. \\ Departments of Neurological Surgery, New York Presbyterian Hospital, Weill Medical College of \\ Cornell University, and Memorial Sloan-Kettering Cancer Center, New York, New York
}

\begin{abstract}
Object. Endoscopic fenestration has been recognized as an accepted treatment choice for patients with symptomatic arachnoid cysts. The success of this procedure, however, is greatly influenced by individual cyst anatomy and location as well as the endoscopic technique used. This review was conducted to assess what variables influence the treatment success for different categories of arachnoid cysts.

Methods. Thirty-three consecutive patients who underwent endoscopic fenestration for treatment of an intracranial arachnoid cyst were identified from a prospective database. The surgical indications and techniques were reviewed, and surgical success rates and patient outcomes were assessed. Specific examples of each cyst category are included to illustrate the technical aspects of endoscopic cyst fenestration.

Endoscopic fenestration of arachnoid cysts was successful when judged by cyst decompression, and symptom resolution was noted in $32(97 \%)$ of 33 cases. The one patient with short-term treatment failure underwent a successful repetition of the operation. There were no surgery-related morbidities or deaths.

Conclusions. Arachnoid cysts are a relatively benign pathological entity that can be managed by performing endoscopically guided cyst wall fenestrations into the ventricular system or cerebrospinal fluid-containing cisterns. Proper patient selection, preoperative planning of endoscope trajectory, use of frameless navigation, and advances in endoscope lens technology and light intensity combine to make this a safe procedure with excellent outcomes.
\end{abstract}

\author{
KEY WORDS • endoscopy • arachnoid cyst • ependymal cyst • fenestration • \\ minimally invasive surgery $\bullet$ children
}

Endoscopic management of intracranial pathological conditions has always posed a unique challenge. Nevertheless, the diversity of pathological entities that are accessible via endoscopy is equaled only by the number of creative approaches neurosurgeons have devised to treat them. The anatomy of the ventricular system lends itself to direct approaches for certain intraventricular lesions, or it can be used as a conduit through which less accessible lesions can be reached. In this report we will review several cystic intracranial pathological entities and discuss those lesions and regions most amenable to endoscopic management, with a focus on the surgical management of arachnoid cysts. Although there is a wide diversity of anatomical locations within which arachnoid cysts are found, we will highlight three common ones: the middle fossa, the septal region, and the suprasellar or prepontine cisterns. We will review the anatomy of and approaches to each region as well as our own experience in the endoscopic management of these challenging lesions.

Endoscopy has been used successfully for decades to treat a variety of pathologies within the central nervous system, including removal of colloid cysts, ${ }^{1,5,11}$ biopsy sampling and removal of intraventricular brain tumors, ${ }^{2,20,21}$ treatment of obstructive hydrocephalus ${ }^{10}$ and management of intracranial cysts. ${ }^{19,22}$ Using the conduits of the ventricular system for endoscopic access to lesions such as colloid

Abbreviations used in this paper: $\mathrm{BA}=$ basilar artery; $\mathrm{CSF}=$ cerebrospinal fluid; $\mathrm{MR}=$ magnetic resonance. cysts or intraventricular tumors is intuitive. Other cystic lesions, including arachnoid, septum pellucidum, porencephalic, and pineal cysts, and cysts from multiloculated hydrocephalus, are equally favorable to treat endoscopically, because of their position adjacent to subarachnoid or intraventricular spaces. In this review, however, we will focus exclusively on arachnoid cysts and their management with endoscopic technology.

\section{CLINICAL MATERIAL AND METHODS}

\section{Patient Selection}

Between 1998 and 2005, we performed surgery for 33 consecutive intracranial cysts by using endoscopic or endoscopically assisted cyst fenestration (Table 1). Of these lesions, 25 could clearly be defined as developmental arachnoid cysts. Eight additional patients with diencephalic, mesencephalic, or porencephalic cysts were included in the case series because their surgical indications, procedures, and goals were similar. Patients were excluded from our study if the cyst was a tumor-related one or if it was primarily associated with multicompartmentalized hydrocephalus secondary to meningitis, even if the procedures were often identical to the arachnoid cyst fenestration. The pathophysiological features of those cysts are clearly distinct from those involved in arachnoid cyst formation, and these patients are prone to recurrent formation of their lesions.

The age range of the patients was 1 week to 58 years, and 
TABLE 1

Demographic data, lesion location, and success of endoscopic fenestration in 33 consecutive patients with intracranial cysts*

\begin{tabular}{|c|c|c|}
\hline Case No. & Age, Sex $\dagger$ & Region of Arachnoid Cyst \\
\hline 1 & 32 yrs, $M$ & cavum septum pellucidum \\
\hline 2 & $1 \mathrm{wk}, \mathrm{M}$ & cavum septum pellucidum \\
\hline 3 & 51 yrs, F & cavum septum pellucidum \\
\hline 4 & $20 \mathrm{yrs}, \mathrm{M}$ & cavum septum pellucidum \\
\hline 5 & 32 yrs, M & cavum septum pellucidum \\
\hline 6 & 2 yrs, $M$ & velum interpositum \\
\hline 7 & $5 \operatorname{mos}, \mathrm{F}$ & velum interpositum \\
\hline 8 & 31 yrs, F & cavum vergae \\
\hline 9 & 58 yrs, F & pineal region \\
\hline 10 & $2 \operatorname{mos}, \mathrm{M}$ & quadrigeminal plate \\
\hline 11 & $16 \operatorname{mos}, \mathrm{F}$ & suprasellar \\
\hline 12 & 2 yrs, F & suprasellar \\
\hline 13 & $1 \mathrm{yr}, \mathrm{M}$ & suprasellar \\
\hline 14 & 44 yrs, M & suprasellar \\
\hline 15 & $3 \operatorname{mos}, \mathrm{M}$ & suprasellar \\
\hline 16 & $2.5 \mathrm{yrs}, \mathrm{M}$ & suprasellar \\
\hline 17 & $10 \mathrm{yrs}, \mathrm{M}$ & suprasellar \\
\hline 18 & 9 yrs, F & temporal fossa \\
\hline 19 & $7 \mathrm{mos}, \mathrm{M}$ & temporal fossa \\
\hline 20 & 4 yrs, M & temporal fossa \\
\hline 21 & 2 wks, M & intraventricular \\
\hline 22 & 2 yrs, M & parietal intracerebral \\
\hline 23 & $3 \operatorname{mos}, \mathrm{M}$ & frontal intracerebral \\
\hline 24 & 21 yrs, F & porencephalic cyst \\
\hline 25 & 38 yrs, M & porencephalic cyst \\
\hline 26 & $51 \mathrm{yrs}, \mathrm{M}$ & porencephalic cyst \\
\hline 27 & 58 yrs, $\mathrm{F}$ & interhemispheric \\
\hline 28 & $10 \mathrm{yrs}, \mathrm{M}$ & interhemispheric \\
\hline 29 & 2 yrs, $M$ & isolated 4th ventricle \\
\hline 30 & $1 \mathrm{wk}, \mathrm{F}$ & posterior fossa/Dandy-Walker \\
\hline 31 & 43 yrs, F & mescencephalic cyst \\
\hline 32 & 5 yrs, $M$ & diencephalic cyst \\
\hline 33 & 23 yrs, $\mathrm{F}$ & diencephalic cyst \\
\hline
\end{tabular}

* All but the patient in Case 6 (who underwent a repeated operation) were shunt free, for a $96.9 \%$ success rate.

$\dagger$ The male/female ratio was $21: 12$.

these individuals were disproportionately male, with 21 male and 12 female patients. There were 21 children and 12 adults in the cohort. Of the most commonly diagnosed regions, none of the patients with temporal fossa cysts and only one with a prepontine/suprasellar region cyst was an adult. The most common reason for seeking medical attention in adults was headache (seven patients) and in the pediatric population it was macrocrania (seven). Four children were recognized to harbor intracranial cysts on prenatal imaging. A wide range of other predictable yet less common diagnoses, including panhypopituitarism from a suprasellar lesion, to ocular symptoms and gait disturbance from a suprasellar lesion, were included in the patient series.

Because of their often asymptomatic presentation and their benign pathological features, the optimal management for arachnoid cysts may range from conservative observation to surgical intervention. The most favorable surgical candidates are chosen based on two major criteria: symptomatology and lesion location. Patients who are asymptomatic are generally poor surgical candidates. In some circumstances, extremely large asymptomatic lesions, especially in growing children, may be more aggressively approached based on the rationale that decompression may help prevent developmental delays. In the adult population, surgical management of asymptomatic lesions must have a more rigorous justification, such as evidence on neuroimaging of progressive growth or early obstructive hydrocephalus.

With respect to lesion location, almost all arachnoid cysts can be managed surgically, but not all are optimal candidates for endoscopy. Choosing patients whose cyst is in close communication with a ventricle or a cisternal compartment will increase the likelihood of establishing an effective fenestration endoscopically. If this criterion cannot be met, cystoperitoneal CSF shunting may provide greater long-term decompression of the cyst. As an example, in Fig. 1, $\mathrm{T}_{2}$-weighted MR images obtained in a patient with a large middle fossa cyst are shown. Although cysts in this category generally make excellent candidates for endoscopic fenestration, in this case a cortical mantle is present between the suprasellar cistern and the cyst wall (arrow), precluding the safe creation of a cystocisternotomy. To summarize, symptomatic patients with arachnoid cysts contiguous to a CSF-containing space make excellent candidates for endoscopic treatment.

\section{Equipment and Technique}

All procedures were performed after induction of general anesthesia. No seizure prophylaxis or steroid therapy was used. All patients received intravenous antibiotic prophylaxis before skin incisions were made. To begin the procedure, the patient's skull was secured with pin fixation if frameless neuronavigation was being used. A burr was used for access, with the entry site determined by the region within which the arachnoid cyst was located. A $0^{\circ}$ or $30^{\circ}$ rigid lens endoscope (MINOP; B. Braun Aesculap, Tuttlingen, Germany) was used for the initial cannulation. The endoscope's sheath has an outer diameter of $6 \mathrm{~mm}$ and a 2-mm working channel. When stereotaxy was used for trajectory planning, the sheath was registered using an optically guided stereotactic system (Stealth; Medtronic, Inc., Minneapolis, MN). Planning the trajectory is often the most crucial aspect of the surgical procedure. An entry site must be selected that provides a direct, linear route to the cyst to accomplish two distinct goals. One is to minimize any torque on the cortical or intraventricular neural tissue. The second is to allow direct, inline access to the distal edge of the cyst wall; the edge abutting the CSF-containing cistern into which the cyst will be fenestrated.

Fenestration of the arachnoid cyst into the cisternal or ventricular compartments must be done only after thorough inspection of the cystic surface. Continuous irrigation is used during endoscopy to maintain a clear medium for image transmission and for hemostasis. A constant purge is also maintained to avoid overinsufflation of the cystic lesion or ventricular compartment, inadvertently leading to raised intracranial pressure. The continuous irrigation also provides real-time feedback about the translucency and consistency of the membranes onto which the fluid current is directed, allowing for the safe selection of fenestration target sites.

Once the target site has been selected, a bipolar diathermy instrument is used both to attenuate the tissue and to provide a blunt instrument with which to perforate the cyst wall. After a small communication between the cyst and the cistern or ventricle has been made, a progressive enlargement of the fenestration is created using a combination 


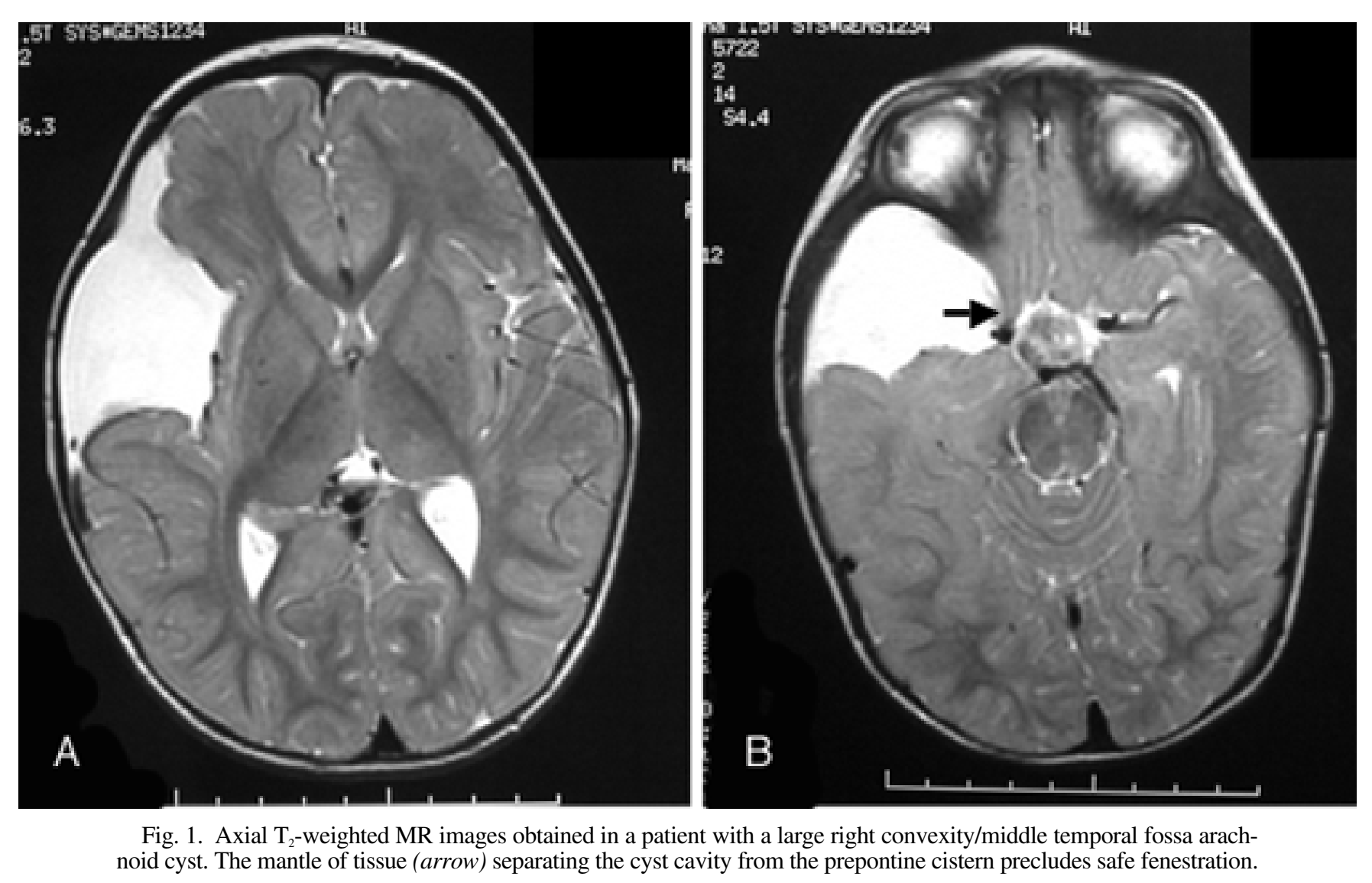

of balloon dilation, blunt perforation, and sharp scissors to connect a series of smaller fenestrations and create a large conduit for CSF egress. The combination of balloon tamponade with a No. 3 French embolectomy catheter, bipolar diathermy, and continuous irrigation provide sufficient hemostasis given the relatively avascular fenestration surfaces. At the completion of the procedure, irrigation is briefly withheld, and the adequacy of the cyst fenestration can be judged by the to-and-fro motion of the free edges of the fenestrations, which should move freely with the CSF pulsations of the cardiac cycle if they are in adequate communication with a CSF-containing compartment.

\section{REGIONAL CONSIDERATIONS}

\section{Middle Cranial Fossa}

The most common location for arachnoid cysts is the middle cranial fossa. ${ }^{6,17,24}$ There is still substantial support for the use of open or "keyhole-approach" craniotomy in this particular location, ${ }^{14}$ compared with other intracranial regions where endoscopy has been more uniformly adopted. Proponents of craniotomy point to the larger working space it affords, leading to better hemostasis; however, in other case series investigators have suggested equal success rates for neuroendoscopic management of middle fossa cysts. ${ }^{12}$

With respect to the endoscopic surgical approach to middle cranial fossa cysts, a burr hole is placed on the coronal suture approximately 5 to $6 \mathrm{~cm}$ lateral to midline to avoid vessels of the sylvian fissure and to allow an optimal trajectory to the medial edge of the cyst and the target cistern. Once the cyst is safely cannulated, the anatomy of the region dictates the placement of the distal fenestration. By advancing the endoscope along the sphenoid ridge, the internal carotid artery bifurcation and the ipsilateral optic apparatus should be visible. The approach to fenestration again uses a combination of blunt perforation of translucent membranes, followed by progressive dilation of the stoma through multiple possible mechanisms. This creation of a fenestration between the cyst and the basal cisterns is called a cystocisternotomy. Postoperative neuroimaging sometimes indicates a reduction in cyst size, but resolution of symptoms is the most reliable means with which to assess outcome.

\section{The Septal Region}

The septum pellucidum is composed of two thin walls that are normally opposed to one another. It is attached from above to the corpus callosum, and below to the fornix. Cysts of the septum pellucidum result from a defect in the hemispheric cleavage. ${ }^{8}$ Embryologically, the walls of the septum pellucidum are not opposed and the resulting cavity is referred to as the cavum septum, which communicates with another one, the cavum vergae. These cavities do not communicate with the ventricular system; rather, they gradually close in a caudal-to-rostral fashion and should not be evident within several months of birth. Nevertheless, persistence of one of these cavities has been noted in approxi- 
mately $1 \%$ of postnatal computerized tomography scans. ${ }^{3}$ Despite this relative frequency, the majority of patients in whom these septal region cysts are discovered remain asymptomatic for life. Symptomatic cysts are infrequent but have been recognized and treated surgically for decades. ${ }^{4}$ Only recently, however, has endoscopy been added to the armamentarium of approaches to this region. ${ }^{7,8,12,15}$

These cysts become symptomatic by causing obstruction of the foramen of Monro or by compression of midline structures. The symptomatology, aside from headache, is often subtle, because emotional and memory disturbances are nearly always encountered as part of the clinical scenario,${ }^{15}$ presumably due to the cysts' proximity to the fornix and limbic system-associated pathways. Visual disturbances, hemiplegias, and dysesthesias have all been reported, and distortion of vascular structures, compression of the hypothalamoseptal triangle, and compression of visual pathways have all been suggested as contributing factors to these cysts' varied symptomatology.

Endoscopic management is used to establish a patent communication between the cystic midline structure and the ventricular system; this procedure is called a cystoventriculostomy. Two anatomical trajectories can be followed to enter the lateral ventricle: 1) an approach similar to that used when performing an endoscopic third ventriculostomy, on the coronal suture $3 \mathrm{~cm}$ from the midline; or 2) an occipital burr hole to optimize the trajectory into the atrium of the lateral ventricle. Whereas the coronal approach is more familiar to most surgeons, the occipitally oriented one has a large advantage; the ability to approach both leaflets of the cyst perpendicularly.

Once the ventricular system has been cannulated with the introducer sheath, identification of normal ventricular anatomy, including the foramen of Monro, the choroid plexus, and the septal and thalamostriate veins allows for proper orientation. The cavum septum should be identified medially and an avascular site chosen for the fenestration. Once a wide stoma is created, the cavum septum is entered and the neuroendoscope is navigated to the contralateral wall, which is identically fenestrated from inside the cyst into the contralateral ventricle. While the instrument is on the contralateral side, visualization of the choroid plexus is evidence of successful fenestration into the ventricle.

Although bilateral stoma may be technically reassuring, it is likely that a unilateral fenestration, if of adequate dimension, would decompress a midline cyst of the septum and eliminate the obstruction of both foramina. Postoperative success can be judged by decompression of the cyst and resultant alleviation of the hydrocephalus. The leaflets of the cavum are seen to separate from the ependymal surface of the lateral ventricle, thus establishing decompression.

\section{Suprasellar Region}

Suprasellar arachnoid cysts are rare lesions that constitute approximately 5 to $12.5 \%$ of all arachnoid cysts. ${ }^{6,23}$ Their origin is believed to be the result of a slit valve mechanism created when the BA pierces the prepontine arachnoid membrane. ${ }^{18}$ Because of this origin, the term prepontine arachnoid cyst may be more accurate than suprasellar cyst. A variety of interesting symptomatology has been attributed to large cysts in this region, besides hydrocephalus and its associated spectrum of symptoms. Visual impairments, endocrinopathies, and head bobbing may all be observed in patients presenting with neuroimaging evidence of a prepontine arachnoid cyst. In contrast to septal region cysts, these are only infrequently found incidentally. Similar to the methods used for cysts in other intracranial locations, a variety of surgical techniques have been used to treat these challenging cysts, including open fenestration, cyst resection, cystoperitoneal shunts, and stereotactic aspirations. In several reports, investigators have now advocated the use of endoscopy for the management of cysts in this region. ${ }^{9}{ }^{13,16}$ In 2004, Wang, et al., ${ }^{23}$ reported on six patients who underwent endoscopic ventriculocystocisternotomy for management of symptomatic prepontine arachnoid cysts, adding endoscopy to the list of surgical options.

Planning the precise entry site is crucial when endoscopy is being attempted in prepontine cysts. A trajectory is selected, such that the endoscope will facilitate fenestration at both apical and basal cyst membranes, without placing any torque in a rostral or caudal direction. The entry site is roughly at the coronal suture, 1 to $2 \mathrm{~cm}$ off the midline. The endoscope is directed into the lateral ventricle and standard anatomical landmarks are used to advance it through the foramen of Monro from the right lateral ventricle into the third ventricle. Once through the foramen, identification of the blue, apical dome of the arachnoid cyst is typically accomplished, followed by creation of a ventriculocystostomy by using bipolar diathermy and sharp dissection. There is no need to resect the membrane at this rostral extent. The endoscope is then advanced into the lesion to the basal cyst membrane, where a cystocisternotomy is performed without the use of any energy source. This can be accomplished using blunt biopsy forceps to create fenestrations, typically between cranial nerves exiting the brainstem.

Postoperative assessment can be performed most accurately with MR imaging. Specifically, $\mathrm{T}_{2}$-weighted, fluid-attenuated inversion-recovery, and cine MR sequences all are useful in confirming patency of fenestration sites and pulsatility of flow through them. In addition, the cyst's volume can be determined and pre- and postoperative measurements can be compared. The radiographic features found consistently in prepontine arachnoid cysts include vertical deflection of the optic chiasm and mammillary bodies and an effacement of the ventral pons. In the case series of suprasellar arachnoid cysts managed endoscopically by Wang, et al., ${ }^{23}$ preoperative symptoms had improved in all patients after a mean follow-up duration of 26 months, and no further fenestrations or CSF diversions were required once the fenestration had been completed. In addition, these authors demonstrated that the displaced optic apparatus and mammillary bodies returned to a more horizontal position and pontine deformation improved.

\section{RESULTS}

Of the 33 patients, only one had to return to the operating room for a related procedure. Successful fenestration has therefore been $96.9 \%$. There were no procedure-related morbidities and no deaths. The one patient with a failed fenestration was a 3-year-old boy who had missed his developmental milestones and was found to be hydrocephalic due to a large central midline cystic structure caus- 

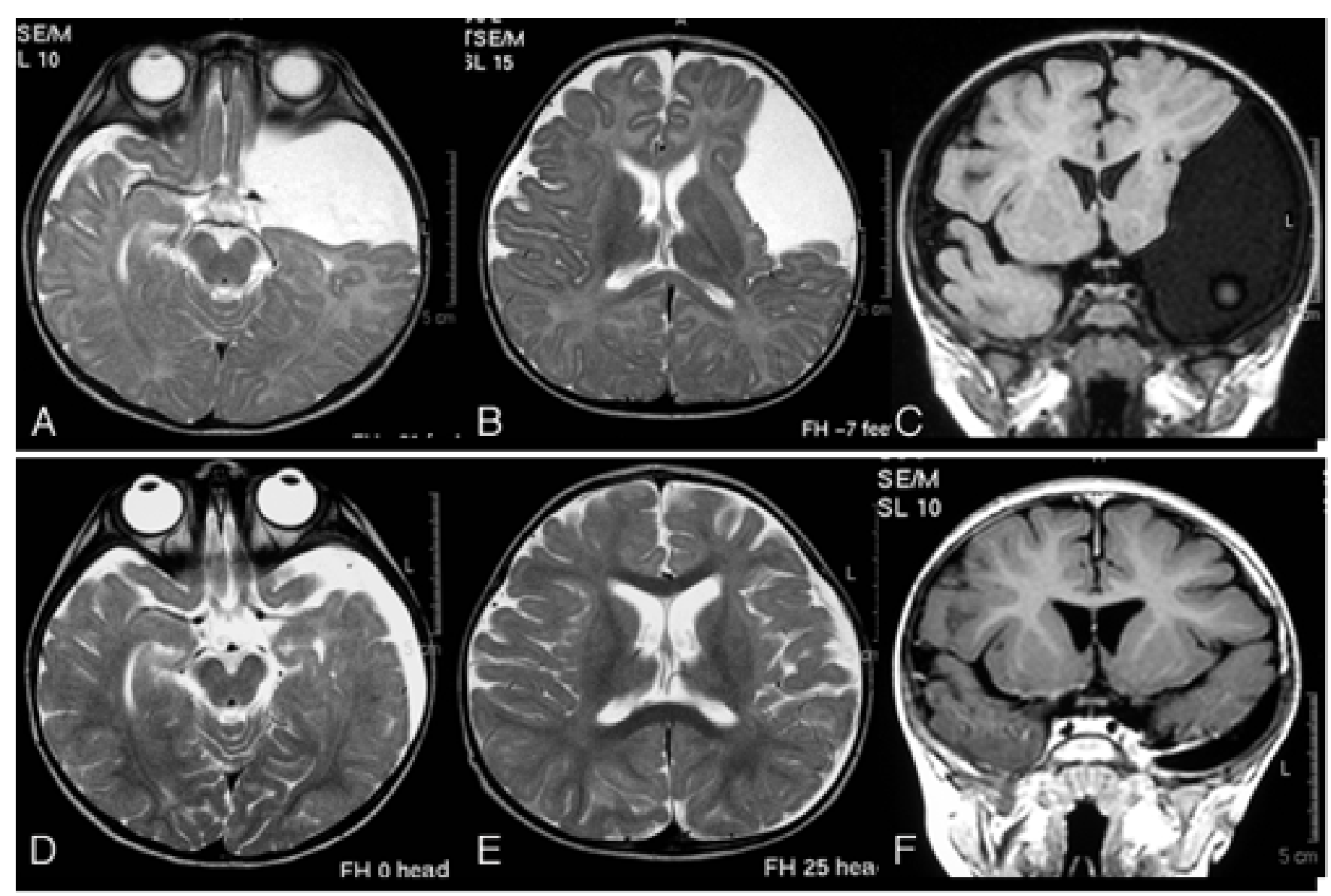

Fig. 2. Case 19. Preoperative (A and B) and postoperative (D and E) axial $\mathrm{T}_{2}-$ weighted and preoperative (C) and postoperative $(\mathrm{F})$ coronal $\mathrm{T}_{1}$-weighted $\mathrm{MR}$ images obtained in a patient with endoscopic fenestration of a right middle cranial fossa arachnoid cyst. The postoperative MR images obtained several months after surgery demonstrate dramatic improvement of cyst volume and temporal lobe effacement.

ing obstruction at the level of the midbrain. He underwent an endoscopic third ventriculostomy and cyst fenestration, with subsequent evaluations revealing a decrease in the size of the cyst and a functional third ventriculostomy. After 3 months, however, repeated imaging revealed that the cyst contents had reaccumulated after the patient was admitted to the emergency department for involuntary motor movements thought to be pressure waves within the velum interpositum cyst. After repeated fenestration, he experienced complete resolution of his cyst and the accompanying hydrocephalus. There was no clear explanation based on the intraoperative findings from either surgery to explain why the fenestration failed.

\section{ILLUSTRATIVE CASES}

\section{Case 19}

History. This 7-month-old baby had been receiving medical attention since presenting as an infant with generalized seizures. At that time, results of neuroimaging studies were consistent with a large Type III middle fossa arachnoid cyst with no hydrocephalus (Fig. 2A-C). Due to the symptomatic nature of the cyst, an endoscopic cyst fenestration was recommended.
Operation. A single burr hole was placed in the temporal squamosa $1 \mathrm{~cm}$ anterior to the external auditory canal. The underlying dura mater was bluish, and it was coagulated and incised and the cyst entered. Navigating the endoscope medially, the tentorial edge, oculomotor nerve, carotid artery, optic nerve, and olfactory nerve could all be identified. These structures were all deeply invested in a semiopaque, pulsatile membrane consistent with the medial wall of the arachnoid cyst. Multiple segments of this membrane that are completely devoid of neurovascular structures can be seen. These membranes are opened using a combination of blunt and sharp perforation, followed by balloon dilation and sharp dissection. This created a wide stoma between the tentorium and the oculomotor nerve, and between the oculomotor nerve and carotid artery.

Advancing the endoscope to the new fenestration, visualization through the contralateral side of the cyst to the prepontine cistern surface can be accomplished. Successful creation of this cystocisternotomy between the middle fossa arachnoid cyst and the prepontine cistern can be confirmed by visualization of the ventral pontine surface, BA, and pituitary stalk. (See Video 1 to view abridged footage of an identical cyst fenestration.)

Video 1. Abridged movie demonstrating key technical fea- 


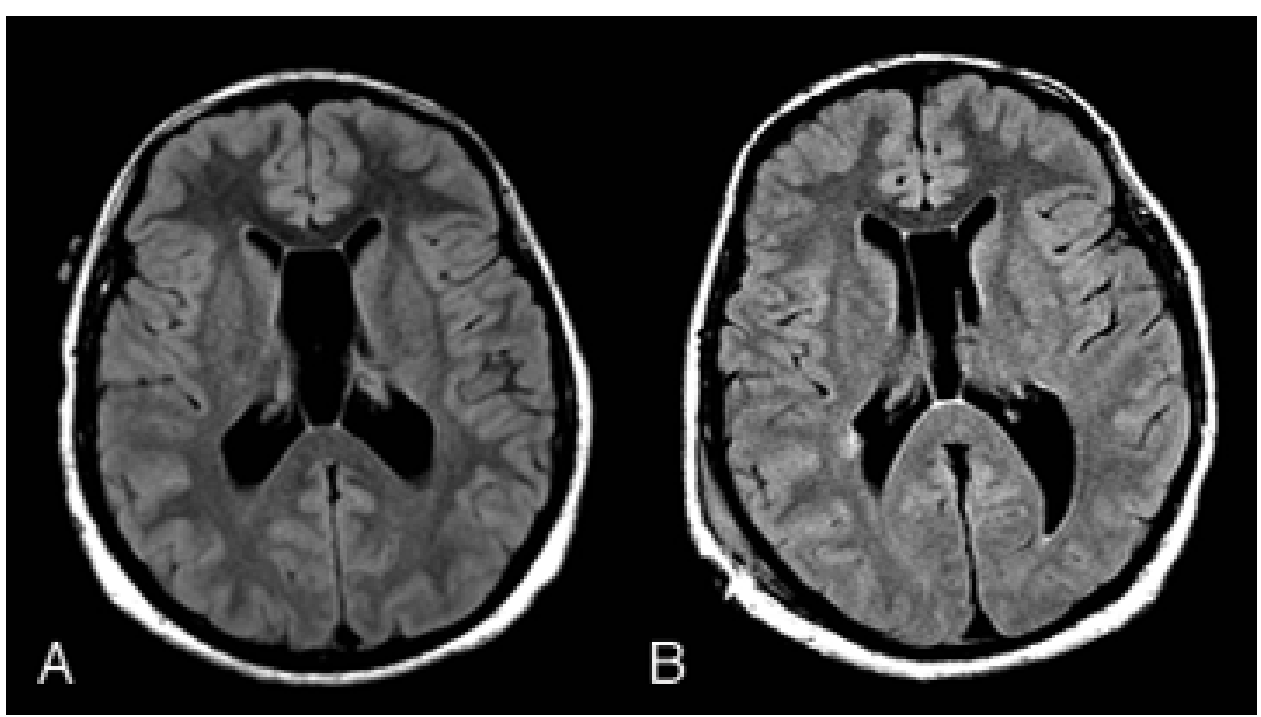

Fig. 3. Case 4. Preoperative (A) and postoperative (B) $\mathrm{T}_{1}$-weighted MR images obtained in a patient with endoscopic fenestration of a cavum septum pellucidum/septal region arachnoid cyst via a right occipital approach. The postoperative MR image was obtained only 2 days after endoscopic fenestration, and resolution of both cyst size and ventriculomegaly are evident.

tures of an endoscopic fenestration of a middle fossa arachnoid cyst into the prepontine cistern. For more details, see description of the procedure for Illustrative Case 1. (Click here to view with Windows Media Player and a broadband connection, or here to view with RealPlayer.)

Neuroimaging Findings and Postoperative Course. Postoperative MR images (Fig. 2D-F) revealed a dramatic resolution of the cystic dimensions, and mass effect on the adjacent cortex.

\section{Case 4}

History. This 20-year-old man received a diagnosis of trisomy-21 at birth. He only recently came to neurosurgical attention because of progressive headaches and subtle cognitive changes over a period of several months. As part of his workup, an MR imaging study of the brain revealed ventriculomegaly involving both lateral ventricles and a large midline cyst consistent with cavum septum pellucidum and cavum vergae (Fig. 3A). Treatment options were discussed with the family and they agreed to authorize endoscopic fenestration of the cyst.

Operation. The patient's head was secured with threepoint fixation and, using stereotactic guidance, an entry site was selected in the right occipital region to optimize the trajectory into both leaves of the cavum septum pellucidum and vergae. Using a 6-mm rod lens endoscope integrated into the frameless stereotactic navigational system, the endoscopic sheath was passed into the right atrium of the lateral ventricle. The medial aspect of the ventricle had been replaced by a large, bulbous, cystic structure that had a blue, attenuated wall. The foramen of Monro could not be identified. Large fenestrations were created in an avascular area of the cyst wall; the endoscope was navigated into the cavum, after which the contralateral leaflet was fenestrated in a similar manner before removing the endoscope.

Neuroimaging Findings and Postoperative Course. Post- operative MR imaging revealed decompression of the cavum septum and resolution of the obstructive hydrocephalus (Fig. 3B).

\section{Case 12}

History. This 2-year-old girl had recently received a diagnosis of divergent macrocrania with delayed development. An evaluation by a pediatric neurologist was performed, and it revealed global delay in reaching milestones but no other significant neurological findings focally. An MR image of the brain was obtained, revealing severe ventricular dilation with noncommunicating hydrocephalus and a large cystic mass in the suprasellar/prepontine cisterns. The neuroimaging findings were consistent with a prepontine arachnoid cyst causing obstructive hydrocephalus (Fig. 4). Treatment options were discussed with the family, and they agreed to let their child undergo endoscopic fenestration of the cyst.

Operation. A single burr hole was placed and slightly enlarged using rongeurs near the right coronal suture, 1 to $2 \mathrm{~cm}$ off the midline. A rigid endoscope was passed into the right frontal horn of the lateral ventricle, revealing a large cystic mass with a semitranslucent, blue membrane obstructing the foramen of Monro. A combination of bipolar electrocautery and sharp dissection was used to create a large fenestration in this region of the cyst. The endoscope was then navigated into the lesion, where the infundibular stalk, dorsum sellae, both internal carotid arteries and posterior communicating arteries as well as the ventral surface of the brainstem could be identified. The endoscope was navigated into the prepontine cistern, where the bilateral abducent nerves and BA were identified. The membrane at the basal surface of the cyst was identified and blunt dissection was used to create a fenestration in front of the BA between the dural surface of the clivus and the ventral surface of the brainstem. This was enlarged using a No. 

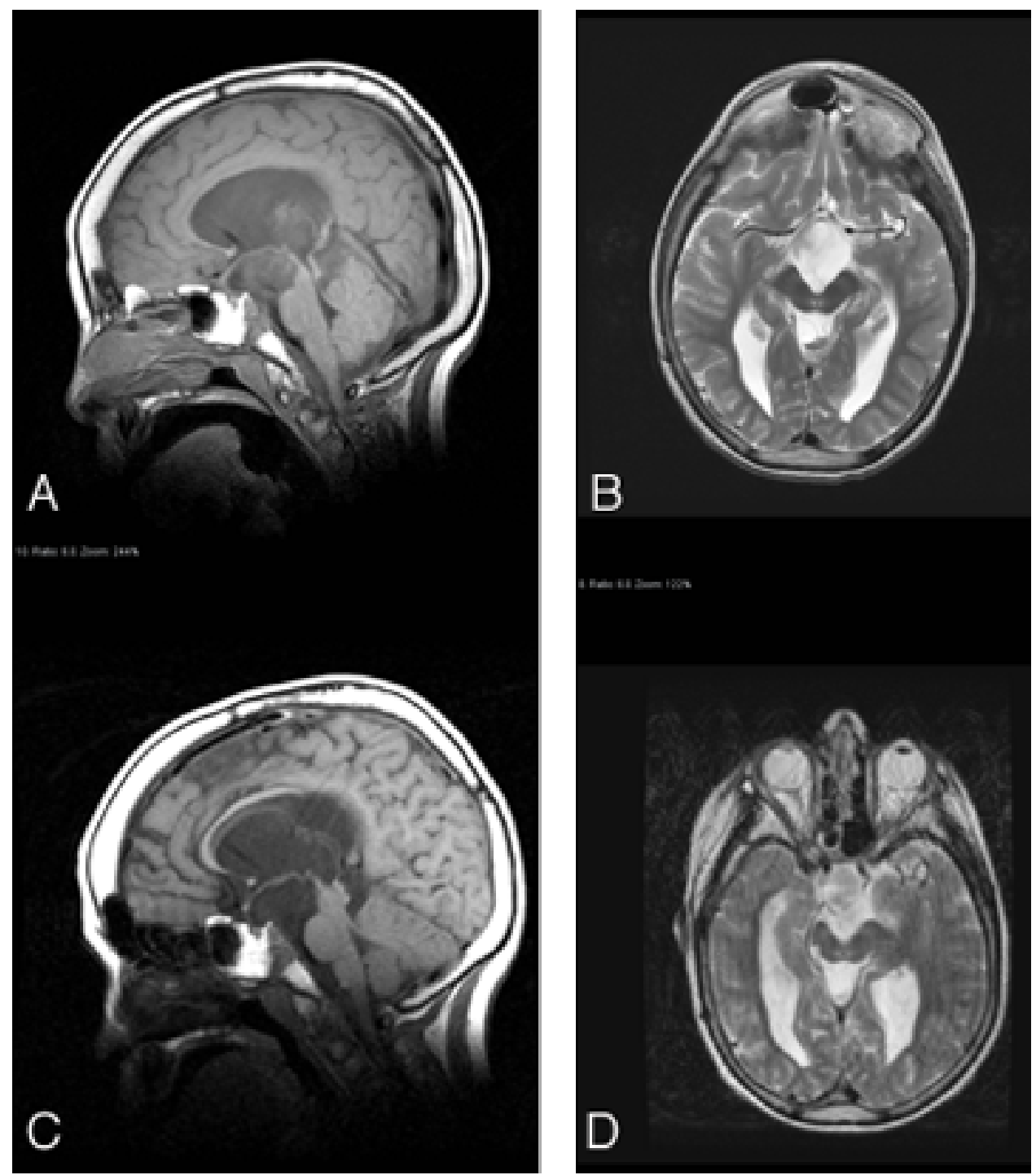

Fig. 4. Case 12. Preoperative (A) and postoperative (C) $\mathrm{T}_{1}$-weighted sagittal and preoperative (B) and postoperative (D) $\mathrm{T}_{2}$-weighted axial MR images obtained in a patient with endoscopic fenestration of a prepontine/suprasellar region arachnoid cyst. The postoperative MR image was acquired 1 week after endoscopic fenestration was completed via a right frontal approach. Less effacement of the diencephalon and a more horizontal position of the optic chiasm are seen. Decompression of the cyst and less mass effect on the mescencephalon are evident.

3 French embolectomy catheter. The membrane became pulsatile with excursions of the cardiac cycle and it was believed that a complete fenestration of the cyst had been achieved.

Neuroimaging Findings and Postoperative Course. Postoperative MR imaging revealed decompression of the prepontine cyst and improvement of the obstructive hydrocephalus (Fig. 4C and D).

\section{DISCUSSION}

With advances in neuroimaging, a large number of asymptomatic arachnoid cysts are being routinely discovered. The management of these lesions in asymptomatic patients remains somewhat controversial, especially in pediatric populations where avoidance of developmental delays or endocrinological or visual abnormalities may outweigh the risk of surgery in an asymptomatic child. Conservative management in asymptomatic adults is less controversial. When surgery is deemed appropriate, a wealth of experience is now available to guide the appropriate surgical approach.

Arachnoid cysts are intraarachnoid CSF-containing lesions that are not in direct communication with the ventricular system. They constitute approximately $1 \%$ of intracranial masses, with 50 to $60 \%$ occurring in the middle cranial fossa. Due to their preponderance in the pediatric population, they are believed to arise as developmental anomalies, although they are not normally associated with other such anomalies of the central nervous system. Arachnoid cysts are often incidental neuroimaging findings, and many pa- 
tients remain asymptomatic. When the cysts become symptomatic, they can cause headaches, increase in head circumference, developmental delay in younger children, visual loss and precocious puberty if located in the suprasellar cistern, or seizures and focal neurological deficits if more cortically centered.

Cystic lesions within the arachnoid membrane are histologically classified as either arachnoid connective tissue or glioependymal tissue. These two broad categories can be found almost anywhere along the neuraxis, although lesions located along the cerebral convexity and spinal cord are mostly arachnoid cysts, whereas those in the supracollicular or retrocerebellar region might be in either pathological class. Pathological examination reveals the derivation of the cyst walls to be from a splitting of the arachnoid membrane, with an inner and outer leaflet surrounding the cyst cavity, but no epithelial lining. In addition, the cyst wall is normally devoid of vasculature. Glioependymal cysts, in contrast, may have an epithelial lining; they are thought to be of neuroectodermal origin.

Arachnoid cysts arise from a gradual expansion of clefts or duplications in or adjacent to normal arachnoidal cisterns. The cysts expand when CSF pulsations, which are concordant with the cardiac cycle, become entrapped between the leaflets of the membrane. It thus follows that their usual contents are indistinguishable from normal CSF, although occasionally cysts with more highly proteinaceous contents can be identified. In contrast to these developmentally acquired cysts, a separate category of acquired arachnoid cysts exists, in which the pathological entity develops secondary to trauma, surgery, or infections. These acquired lesions are also referred to as leptomeningeal cysts, and are similarly thought to arise from violations of one of the arachnoid membranes and a ball valve-like mechanism of CSF pulsation, which favor gradual expansion of the cyst.

Arachnoid cysts occur in multiple locations throughout the intracranial compartment. We have highlighted three common regions (the middle fossa, the septal region, and the prepontine cistern) as locations amenable to endoscopic cyst fenestration, but arachnoid cysts can also occur in the posterior fossa, the cerebellopontine angle, interhemispherically, along the quadrigeminal plate, and anywhere along the cortical surface. In addition, a large array of other cystic lesions is amenable to endoscopy: cysts associated with tumors, such as craniopharyngiomas; or cysts amenable to decompression through an attenuated ventricle wall (for example, diencephalic mass); and those secondary to trauma or surgical cavities (porencephalic cysts), or infections (loculated multicystic hydrocephalus).

Historically, arachnoid cysts have been treated successfully by using cystoperitoneal CSF shunting or open craniotomy for cyst fenestration. Although these were often successful, avoiding a permanent shunt has obvious advantages, such as removing the possibility of obstructions, infections, and surgery-related morbidity such as hemorrhage and catheter misplacement. Craniotomies have their own associated morbidities that are not typically incurred when performing a burr hole opening for an endoscopic cyst fenestration. Reports of endoscopic cyst fenestration were rare just 5 years ago, but the field has since seen a large increase in the number of surgeons who are comfortably and routinely using the endoscope to approach these lesions. In ad- dition, combined procedures, especially in the middle cranial fossa, in which small craniotomies are made for exposure, followed by endoscopic cyst wall fenestrations, are becoming the standard of care. The best procedure remains the one in which the cyst can be fenestrated successfully and safely by a given surgeon.

There remain operator-based preferences, including the use of rigid or flexible endoscopes. We prefer the rigid endoscope for its superior optics, and we change the degree of the endoscope when it is necessary to see difficult angles rather than use the steerable device. Some surgeons routinely use a low-wattage laser system to incise the cyst wall or shrink the lesion to smaller dimensions. We prefer to use only bipolar diathermy and blunt dissection on the cyst wall, and we never use any heat source near any neural or vascular structures (such as in the opticocarotid recess or prepontine cistern), thereby removing any potential for transmitted heat injury.

The rate of long-term patency of our endoscopic fenestrations is excellent. We have required only one return to the operating room for a repeated fenestration. There have been no hemorrhages necessitating aborting the procedure and no procedure-related complications. The MR imaging modality has become an excellent tool through which to judge the success of CSF flow through fenestrations and overall cyst volume reduction. The $\mathrm{T}_{2}$-weighted and fluidattenuated inversion-recovery sequences should be routinely used as part of postoperative evaluations.

\section{CONCLUSIONS}

Although arachnoid cysts are a relatively benign pathological entity, preemptive treatment in children, or in patients with symptomatic lesions regardless of age, can be managed with endoscopically guided cyst wall fenestrations into the ventricular system or CSF-containing cisterns. Proper patient selection, preoperative planning of endoscope trajectory, use of frameless navigation, and advances in endoscope lens technology and light intensity combine to make this a relatively safe procedure with excellent outcomes.

\section{References}

1. Abdou MS, Cohen AR: Endoscopic treatment of colloid cysts of the third ventricle. Technical note and review of the literature. J Neurosurg 89:1062-1068, 1998

2. Badie B, Brooks N, Souweidane MM: Endoscopic and minimally invasive microsurgical approaches for treating brain tumor patients. J Neurooncol 69:209-219, 2004

3. Bogdanoff B, Natter HM: Incidence of cavum septum pellucidum in adults: a sign of boxer's encephalopathy. Neurology 39:991-992, 1989

4. Dandy W: Congenital cerebral cysts of the cavum septi pellucidi (fifth ventricle) and cavum vergae (sixth ventricle): diagnosis and treatment. Arch Neurol Psychiatry 25:44-46, 1931

5. Decq P, Guerinel C, Brugieres P, et al: Endoscopic management of colloid cysts. Neurosurgery 42:1288-1296, 1998

6. Fewel M, Levy M, McComb J: Surgical treatment of 95 children with 102 intracranial arachnoid cysts. Pediatr Neurosurg 25:165-173, 1996

7. Fratzoglou M, Grunert P, Leite dos Santos A, et al: Symptomatic cysts of the cavum septi pellucidi and cavum vergae: the role of endoscopic neurosurgery in the treatment of four consecutive cases. Minim Invasive Neurosurg 46:243-249, 2003 
8. Gangemi M, Maiuri F, Cappabianca P, et al: Endoscopic fenestration of symptomatic septum pellucidum cysts: three case report with discussion on the approaches and technique. Minim Invasive Neurosurg 45:105-108, 2002

9. Golash A, Mitchell G, Mallucci C, et al: Prenatal diagnosis of suprasellar arachnoid cyst and postnatal endoscopic treatment. Childs Nerv Syst 17:739-742, 2001

10. Grunert P, Charalampaki P, Hopf N, et al: The role of third ventriculostomy in the management of obstructive hydrocephalus. Minim Invasive Neurosurg 46:16-21, 2003

11. Hellwig D, Bauer BL, Schulte M, et al: Neuroendoscopic treatment for colloid cysts of the third ventricle: the experience of a decade. Neurosurgery 52:525-533, 2003

12. Kamikawa S, Inui A, Tamaki N, et al: Application of flexible neuroendoscopes to intracerebroventricular arachnoid cysts in children: use of videoscopes. Minim Invasive Neurosurg 44: 186-189, 2001

13. Kirollos R, Javadpour M, May P, et al: Endoscopic treatment of suprasellar and third ventricle-related arachnoid cysts. Childs Nerv Syst 17:713-718, 2001

14. Levy M, Wang M, Aryan H, et al: Microsurgical keyhole approach for middle fossa arachnoid cyst fenestration. Neurosurgery 53:1138-1145, 2003

15. Melikian G, Arutiunov N, Melnikov A: Unusual intraventricular herniation of the suprasellar arachnoid cyst and its successful endosopic management. Minim Invasive Neurosurg 46:113-116, 2003

16. Miki T, Wada J, Nakajima N, et al: Operative indications and neuroendoscopic management of symptomatic cysts of the septum pellucidum. Childs Nerv Syst 21:371-381, 2005

17. Passero S, Filosomi G, Cioni R, et al: Arachnoid cysts of the middle cranial fossa: a clinical radiographical and follow-up study. Acta Neurol Scand 82:94-100, 1990

18. Santamarta D, Aguas J, Ferrer E: The natural history of arachnoid cysts: endoscopic and cine-mode evidence of a slit-valve mechanism. Minim Invasive Neurosurg 38:133-137, 1995

19. Schonherr B, Wolf O, Meier U: Endoscopy of intracranial cysts in the adult patient. Minim Invasive Neurosurg 45: 181-184, 2002

20. Souweidane MM: Endoscopic management of pediatric brain tumors. Neurosurg Focus 18(6a):E1, 2005

21. Souweidane MM, Sandberg DI, Bilsky MH, et al: Endoscopic biopsy for tumors of the third ventricle. Pediatr Neurosurg 33: 133-137, 2000

22. Tirakotai W, Schulte D, Bauer B, et al: Neuroendoscopic surgery of intracranial cysts in adults. Childs Nerv Syst 20: 842-851, 2004

23. Wang J, Heier L, Souweidane MM: Advances in the endoscopic management of suprasellar arachnoid cysts in children. $\mathbf{J}$ Neurosurg (5 Suppl Pediatrics) 100:418-426, 2004

24. Wester K: Peculiarities of intracranial arachnoid cysts: location, sidedness, and sex distribution in 126 consecutive patients. Neurosurgery 45:775-779, 1999

Manuscript received October 29, 2005.

Accepted in final form November 21, 2005.

Address reprint requests to: Mark M. Souweidane, M.D., Department of Neurological Surgery, Weill Cornell Medical College, New York Presbyterian Hospital, 525 East 68th Street, Box 99, New York, New York 10021. email: mmsouwei@med.cornell.edu. 